\title{
Upper limits on the cosmic-ray luminosity of supernovae in nearby galaxies
}

\author{
Rodrigo Sasse $^{a, *}$ and Rita C. Dos Anjos ${ }^{a, b}$ \\ ${ }^{a}$ Universidade Federal da Integração Latino-Americana (UNILA), \\ Programa de Mestrado em Física Aplicada, Av. Silvio Américo Sasdelli, 1842, 85867-670, Foz do \\ Iguaçu, PR, Brazil \\ ${ }^{b}$ Universidade Federal do Paraná (UFPR), \\ Departamento de Engenharias e Exatas, Pioneiro, 2153, 85950-000 Palotina, PR, Brazil \\ E-mail: r.sasse.2020@aluno.unila.edu.br, ritacassia@ufpr.br
}

Interactions between cosmic rays and also between cosmic rays and particles of the Cosmic Microwave Background and the Extragalactic Background Light produce charged and neutral pions. The mechanisms that can produce gamma-ray fluxes associated with cosmic rays are the decay of neutral pions, bremsstrahlung, and inverse Compton scattering from pions. These cascading processes show a correlation between the upper limit on the integral $\mathrm{GeV}-\mathrm{TeV}$ gamma-ray flux and the upper limit on the UHECR luminosity, motivating the study of the multi-messengers to calculate luminosities of UHECRs for specific point sources. We examine the possible sites of ultra-high energy cosmic-ray acceleration in supernovae in nearby galaxies, which were measured by the High Energy Stereoscopic System (H.E.S.S.). The upper limits on the UHECR cosmic-ray luminosity of these sources are calculated with a particular focus on the sources that produce a mixed composition.

$37^{\text {th }}$ International Cosmic Ray Conference (ICRC 2021)

July 12th - 23rd, 2021

Online - Berlin, Germany

\footnotetext{
${ }^{*}$ Presenter
} 


\section{Introduction}

Cosmic rays (CRs) can be accelerated to high energies and travel at almost the speed of light. We know little about their origin, in particular where they come from and how they are accelerated $[1,2]$. Interactions between CRs themselves and also between CRs and particles of the cosmic environment (hadronic interactions) produce charged and neutral pions. The neutron pion decays into two photons, while positrons and electrons are produced due to the decay of charged pions. The three basic mechanisms that can produce gamma-ray fluxes associated with CRs are: i) the decay of neutral pions, ii) electron/positron bremsstrahlung, and iii) inverse Compton scattering of light by electrons/positrons coming from pions. These cascade processes show a correlation between the upper limit on the integral $\mathrm{GeV}-\mathrm{TeV}$ gamma-ray flux and the upper limit on the ultra-high energy CRs (UHECRs) luminosity [3, 4], motivating multi-messenger studies to calculate the luminosities of UHECRs for specific point sources.

The interaction between cosmic rays and the extra-galactic photon background generating multimessenger particles is an important aspect to be studied to get information from the sources. The source BL Lac object TXS 0506+056was found to be active in high-energy gamma-rays and veryhigh-energy gamma-rays with Fermi-LAT and MAGIC telescopes, respectively [5]. Together with observations in gamma-rays, TXS 0506+056is the first observed astrophysical source associated with high-energy neutrinos, detected by the IceCube Observatory - IceCube-170922A [6]. Thus, the production of CRs, gamma-rays, and neutrinos are always interconnected and the complete understanding of the sources and acceleration mechanisms will be possible with a multi-messenger analysis.

\section{Observations and results}

The method was explained in detail in [3, 4]. For both works, the measured upper limit (UL) on the integral flux of $\mathrm{GeV}-\mathrm{TeV}$ gamma-ray was used to constrain the proton and the total (iron) UHECR luminosity. In this paper the SN 2005dn hosted in NGC 6861 is analyzed. Upper limits on the proton and mixed luminosity are set. Studies with additional SNe sources from table 1 is now underway in the presence of extragalactic magnetic fields. We calculate the upper limits on the cosmic-ray luminosity with mixed compositions that fit both measurements on the UHECR energy spectrum and mass composition acquired by Auger Collaboration [7].

Since no gamma-ray measurements were observed from $\mathrm{SNe}$ (table 1), flux upper limits (ULs) have been derived by High Energy Stereoscopic System (H.E.S.S.) at the 95\% confidence level with index $\Gamma=2$ [8], and it is possible to associate the integral flux of $\mathrm{GeV}-\mathrm{TeV}$ gamma-ray measured with UHECR luminosity. We assume an isotropic cosmic-ray emission. The secondary gamma-ray flux is proportional to its cosmic-ray flux or luminosity produced by NGC 6861. Therefore, the production of the gamma-rays is conservative and in function of the cosmic-ray luminosity that may be expressed [4]:

$$
L_{C R}^{U L}=\frac{4 \pi D^{2}\left(1+z_{s}\right)}{\sum_{A} f_{A} \frac{K_{\gamma}^{A}}{\left\langle E_{0}^{A}\right\rangle} \int_{E_{t h}}^{\infty} d E_{\gamma} P_{\gamma}^{A}\left(E_{\gamma}\right)} I_{\gamma}^{U L}\left(>E_{\gamma}^{t h}\right)
$$

where $D_{s}$ is a source at comoving distance from Earth, $A$ is the nuclei composition, $z_{s}$ is the redshift of the source, $\left\langle E_{0}\right\rangle$ is the mean energy of particles in the source, $L_{C R}$ is the total cosmic- 
ray luminosity, $f_{A}$ is the fraction of the total luminosity for composition $A, P_{\gamma}(E)$ is the energy distribution of gamma-rays arriving on Earth and $K_{\gamma}$ is the number of gamma-rays generated from cosmic-ray particle. The calculus taking into account the weight of the point source to the total measured flux by Pierre Auger Observatory.

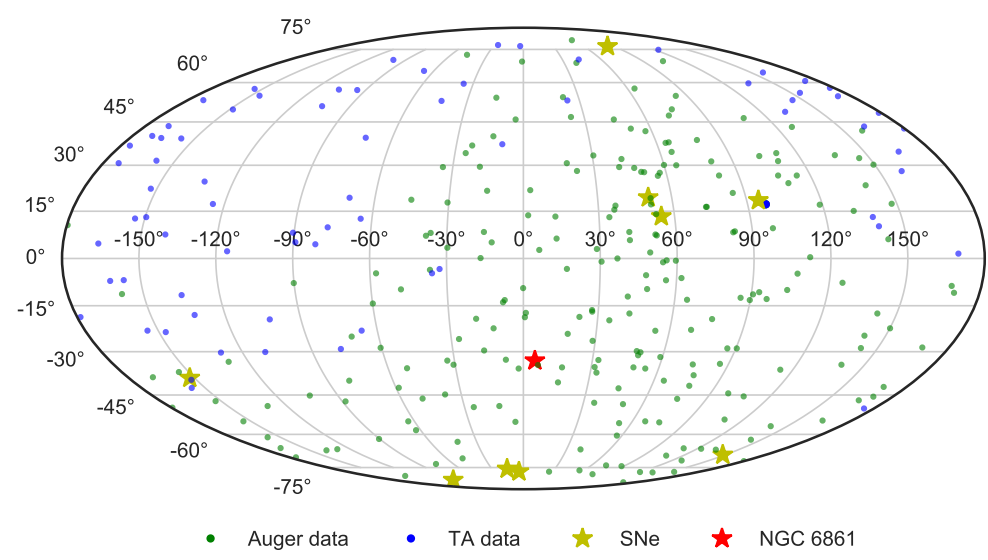

Figure 1: UHECR event locations with $\mathrm{SNe}$ in Galactic coordinates. The green points indicate the arrival directions of 231 events with $E>52 \mathrm{EeV}$ and zenith angle $\theta<80$ from Pierre Auger Observatory [9]. The blue points indicate the arrival directions of 72 events with $E>57 \mathrm{EeV}$ and $\theta<55$ from Telescope Array [10]. The stars indicate the location of nearby galaxies from table 1 .

Table 1: Supernovae parameters observed with H.E.S.S.. The upper limits are computed assuming $95 \%$ confidence level and a power-law index of 2 [8]

\begin{tabular}{|c|c|c|c|}
\hline Host galaxy & SNe & Dist. (Mpc) & $\begin{array}{c}\text { Flux UL } \\
(>1 \mathrm{TeV}) \\
{\left[10^{-13} \mathrm{~cm}^{2} \mathrm{~s}^{-1}\right]}\end{array}$ \\
\hline NGC 7755 & SN 2004cx & $26 \pm 5$. & 1.9 \\
NGC 6861 & SN 2005dn & $38.4 \pm 2.7$ & 0.41 \\
NGC 7793 & SN 2008bk & $4.0 \pm 0.4$ & 4.8. \\
NGC 3095 & SN 2008bp & $29 \pm 6$. & 5.5. \\
NGC 922 & SN 2008ho & $41.5 \pm 2.9$ & 7.7 \\
NGC 175 & SN 2009hf & $53.9 \pm 3.8$ & 5.3 \\
NGC 918 & SN 2009js & $16 \pm 3$ & 11 \\
NGC 4945 & SN 2011ja & $5.28 \pm 0.38$ & 5.2 \\
NGC 4419 & SN 2012cc & $16.5 \pm 1.1$ & 10. \\
NGC 5128 & SN 2016adj & $3.8 \pm 0.1$ & 1.7 \\
\hline
\end{tabular}

The figure 1 shows the sample of supernovae in nearby galaxies and the UHECRs Auger [9] and TA [10] events around the sources. Some SNe are close to the 'hotspots' measured by the Auger and TA [11] and, therefore, the point source UHECRs luminosity of these sources, calculated from the method described, can contribute to the total luminosity of the 'hotspots'. Figure 2 illustrates the 
sources for which we can calculate the UHECR luminosity from integral flux of GeV-TeV gammaray measurement. If the line (proton or iron) is above the measured upper limit of integral flux of gamma-rays then we can obtain an upper limit on the composition luminosity [3, 4]. Finally, the figure 3 presents the show the upper limit on the cosmic-ray luminosity as a function of the spectral index for NGC 6861 hosting SN 2005dn for several compositions at source and for $\mathrm{E}_{\text {cut }}=10^{21} \mathrm{eV}$.

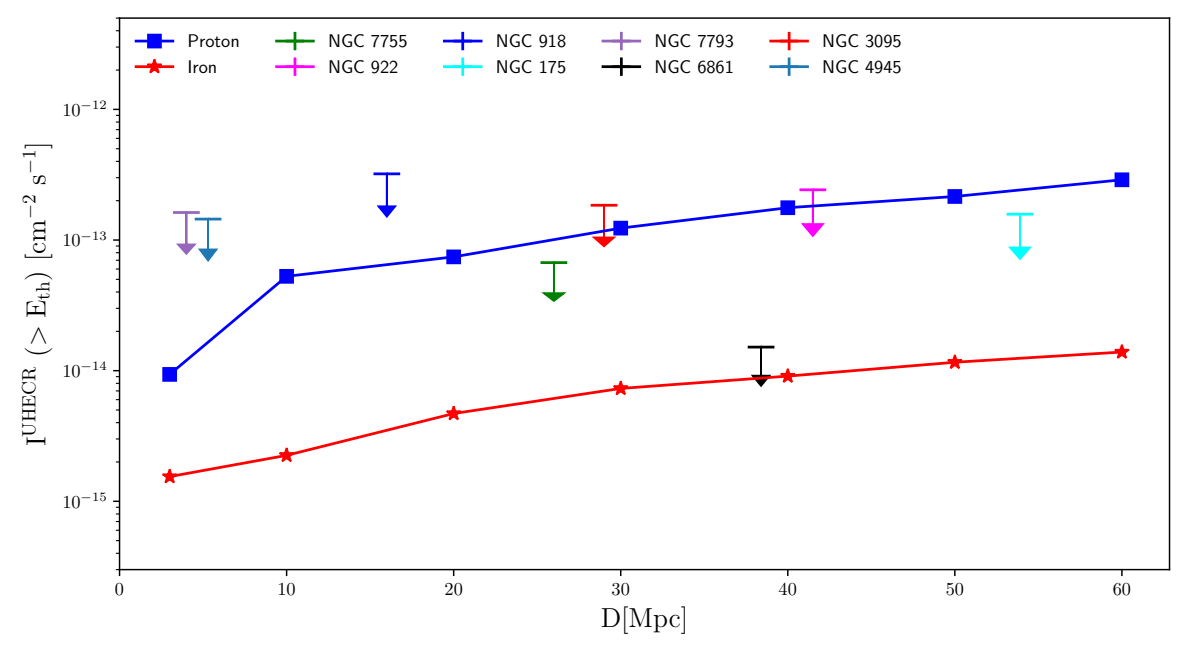

Figure 2: $\mathrm{I}^{\mathrm{UHECR}}$ as a function of the source distance, calculated by using the upper limit on the flux observed by the Pierre Auger Observatory at 95\% CL.



Figure 3: Upper limits on the mixed composition ( $\mathrm{H} ; \mathrm{H}$ and $\mathrm{He} ; \mathrm{H}, \mathrm{He}, \mathrm{N}$ and $\mathrm{Si} ; \mathrm{H}, \mathrm{He}, \mathrm{N}, \mathrm{Si}$ and $\mathrm{Fe}$ ) cosmic-ray luminosity from gamma-rays observations of the source NGC 6861 as a function of the spectral index and for $\mathrm{E}_{\mathrm{cut}}=10^{21} \mathrm{eV}$. 


\section{Conclusion}

Observations of gamma rays establish significant constraints on theories of UHECRs point sources. The upper limits on the UHECR mixed composition luminosity were obtained for the NGC 6861 hosting SN 2005dn. The model shows a direct correlation between the measured upper limit on the $\mathrm{GeV}-\mathrm{TeV}$ integral flux and the secondary gamma-rays from UHECRs propagation using CRPropa3 [12]. The upper limits on the UHECR luminosity for sources of figure 2 will be investigated in different environments in future papers.

\section{Acknowledgments}

We thank our colleagues of the Pierre Auger Collaboration for relevant discussions. R.S. is grateful for the support of UNILA. The research of R.C.A. is supported by Conselho Nacional de Desenvolvimento Científico e Tecnológico (CNPq) grant numbers 307750/2017-5 and 401634/2018-3. She also thanks for the support of L'Oreal Brazil, with partnership of ABC and UNESCO in Brazil. We acknowledge the National Laboratory for Scientific Computing (LNCC/MCTI, Brazil) for providing HPC resources of the SDumont supercomputer, which have contributed to the research results reported within this paper. URL: http://sdumont.lncc.br.

\section{References}

[1] K. Kotera and A.V. Olinto, The Astrophysics of Ultrahigh Energy Cosmic Rays, Ann. Rev.Astron. Astrophys. 49, 119 (2011) [arXiv:1101.4256].

[2] Pierre Auger collaboration, P. Abreu et al., Large scale distribution of arrival directions of cosmic rays detected above $10^{18} \mathrm{eV}$ at the Pierre Auger Observatory, Astrophys. J. Suppl. 203, 34 (2012).

[3] A.D. Supanitsky and V. de Souza, An upper limit on the cosmic-ray luminosity of individual sources from gamma-rayobservations, JCAP12, 023 (2013).

[4] R. C. Anjos, A.D. Supanitsky and V. de Souza, Upper limits on the total cosmic-ray luminosity of individual sources, JCAP07, 049 (2014).

[5] M. Cerruti, A. Zech, C. Boisson, G. Emery, S. Inoue, and J-P Lenain., Leptohadronic singlezone models for the electromagnetic and neutrino emission of TXS0506+056. Monthly Notices of the Royal Astronomical Society: Letters 483(1), L12-L16, 11 (2018).

[6] S. Gao, M. Pohl, and W. Winter. On the direct correlation between gamma-raysand PeV neutrinos from blazars. The Astrophysical Journal 843(2), 109 (2017).

[7] Pierre Auger collaboration, P. Abreu et al., Combined fit of spectrum and composition data as measured by the Pierre Auger Observatory, JCAP04, 038 (2017).

[8] H.E.S.S. Collaboratio, Upper limits on very-high-energy gamma-ray emission from corecollapse supernovae observed with H.E.S.S., A\&A 626, A57 (2019). 
[9] Pierre Auger collaboration, P. Abreu et al., Searches for anisotropies in the arrival directions of the highest energy cosmic rays detected by the Pierre Auger Observatory, Astrophys. J. 804, 15 (2015).

[10] Telescope Array Collaboration, R. U. Abbasiet al., Indications of intermediate-scale anisotropy of cosmic rays with energy greater than $57 \mathrm{EeV}$ in the Northern sky measured with the surface detector of the Telescope Array experiment, Astrophys. J. 790, L21 (2014).

[11] N. Globus et al., Can We Reconcile the TA Excess and Hotspot with Auger Observations?, Astrophys. J. 836, L163 (2017).

[12] R. Alves Batista et al., CRPropa3 - a public astrophysical simulation framework for propagating extraterrestrial ultra-high energy particles, JCAP05, 038 (2016). 